
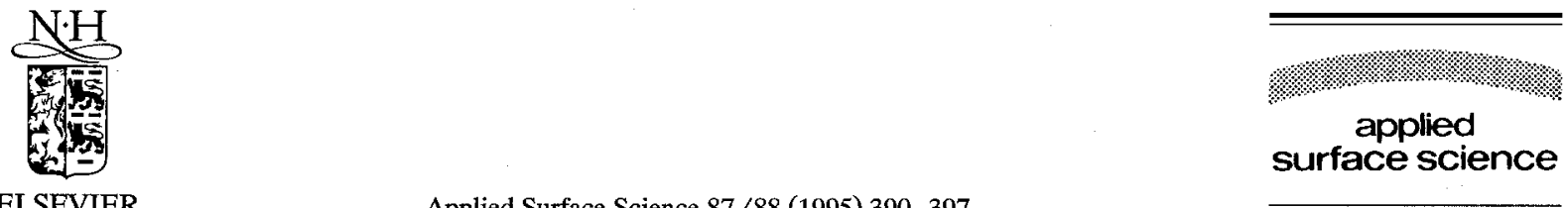

Applied Surface Science 87/88 (1995) 390-397

\title{
Field induced manipulation of fullerene molecules with the STM: a self-consistent theoretical study
}

\author{
M. Devel ${ }^{\mathrm{a}, *}$, C. Girard ${ }^{\mathrm{a}}$, C. Joachim ${ }^{\mathrm{b}}$, O.J.F. Martin ${ }^{\mathrm{c}}$ \\ ${ }^{a}$ Laboratoire de Physique Moléculaire, UA 772 / CNRS Université de Franche-Comté, 25030 Besançon Cedex, France \\ ${ }^{\mathrm{b}}$ CEMES-LOE / CNRS, 29, Rue Jeanne Marvig, B.P. 4347, 31055 Toulouse Cedex, France \\ ${ }^{\circ}$ Swiss Federal Institute of Technology, ETH-Zentrum, CH-8092 Zurich, Switzerland
}

Received 10 July 1994; accepted for publication 10 October 1994

\begin{abstract}
By applying an appropriate voltage between an STM tip and a metallic substrate, it is possible to induce highly localized electrostatic fields. In this paper, it is shown that the apex of the STM probe, responsible for the resolution, confines an electric field of small lateral extension inside the junction. The change in the potential energy of an adsorbate submitted to such a field is calculated with a self-consistent scheme. A microscopic description of both the tip-apex and the adsorbates is used and the correlations between each polarizable centre are accounted for with a discretized Lippmann-Schwinger equation. Several applications show that our real space approach is extremely attractive for studying electrostatic field distributions in low symmetry systems. Field induced manipulation processes of $\mathrm{C}_{60}$ molecules are discussed in this context.
\end{abstract}

\section{Introduction}

Besides its application as an analytical instrument, the scanning tunneling microscope (STM) can also be used to produce new atomic or molecular arrangements stabilized by the atomic corrugations of a surface [1-8]. A large variety of manipulation processes performed on numerous systems has already been described. The simplest method for manipulating adsorbates with an STM exploits the physical interaction between the probe tip and the adsorbed species. The pioneering work of Eigler and coworkers [1], performed on the $\mathrm{Xe}-\mathrm{Ni}(110)$ system, is an impressive demonstration of its feasibility. In this

\footnotetext{
${ }^{*}$ Corresponding author. Fax: +3381666475 .
}

experiment the adsorbate was slid along the surface by applying a lateral interaction or by using the van der Waals trap induced by the tip [9].

The STM devices also offer other interesting possibilities of moving an adsorbate in a controlled way. For an appropriate bias voltage, the presence of a strong electrostatic field confined between the STM tip apex and the substrate can be used to finger and displace the adsorbate. We then deal with the socalled field induced manipulation process extensively described in the literature [7]. Since 1991 there has been a continuous effort to develop and improve this experimental technique. Thus, as described in the recent works of Avouris and coworkers [4], the STM can even be used to manipulate at room temperature strongly bound silicon atoms or clusters on Si surfaces, by applying $3 \mathrm{~V}$ voltage pulses. In similar experiments, field-assisted manipulations 
were demonstrated by Stroscio et al using Cs atoms on $\mathrm{GaAs}$ and $\mathrm{InSb}(110)$ surfaces [6].

In the case of a pure sliding process, the adsorbates are moved along the surface atomic rows without destroying the binding existing between the manipulated species and the substrate. The motion can then be achieved not only by playing on the tip-adsorbate interaction energy $U_{\mathrm{ta}}$, but also by exploiting the electrostatic energy $U_{\mathrm{el}}$ coupling the bias external field and the adsorbate. An advantage of this second procedure lies in the possibility to precisely adjust the threshold tip height corresponding to a sequence of controllable manipulations.

The understanding of the physical mechanisms underlying such field induced manipulation processes is mandatory to precisely handle single atom and molecule [10-12]. A precise calculation of $U_{\mathrm{el}}$ requires specific models adapted to the very complex geometry of the tip-adsorbate-surface junction. The aim of the present paper is twofold. First, we will show from a self-consistent treatment that the last atoms of the apex, responsible for the atomic STM resolution, confine a strong electric field when the tip is at tunnelling distance from the surface. This calculation will be developed within the framework of the electrostatic field propagator method (EFP) [12]. In this scheme, for a given tip-sample configuration, the three-dimensional (3D) field distribution around the apex is derived from the knowledge of the self-consistent field inside the STM tip apex. In a second stage we will show how it is possible to extend this approach in order to account for the presence of a large size adsorbate trapped inside the junction. Finally the knowledge of the entire field distribution inside the system will be exploited to determine a general expression for the electrostatic energy $U_{\mathrm{el}}$ which depends on the mutual interactions between all the partners composing the junction. Field induced manipulation processes of fullerene molecules adsorbed on Au(110) surface will be discussed in this context.

\section{3D-field distribution inside the junction}

Well characterized tips are currently made by the build-up technique [13] or by the deposition technique [14]. Recently, it was demonstrated from a

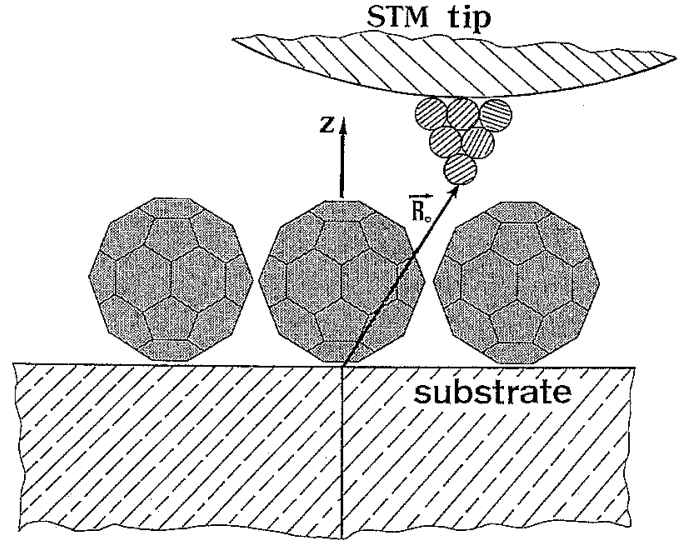

Fig. 1. Schematic illustration of the tip-sample STM junction used for the electric field calculations. The vector $\boldsymbol{R}_{\mathbf{0}}=$ $\left(X_{0}, Y_{0}, Z_{0}\right)$ defines the detector apex position with respect to the surface frame. The shadowed zones schematize the fullerene molecules and the dashed circles represent the atoms of the metallic protrusion.

comparative study between experimental and theoretical STM scans, that the very tip cannot be assimilated to a jellium sphere [12]. In fact, the atomic-size microtip must be considered as a nanometer scale protrusion supported by a larger metallic base [15]. As described in Ref. [12], such a geometry can be handled from a theoretical point of view, by splitting into two parts the physical system: a reference system (in our case the metallic base of the tip plus the surface (cf. Fig. 1)) and the microscopic components localized in the junction (adsorbed molecules plus the metallic probing apex).

The field distribution $\boldsymbol{E}_{0}$ inside the reference system can be obtained by solving the Laplace equation. For example the problem of a sphere or an hyperboloid placed in front of a plane metallic surface has been extensively discussed in the literature [16]. For such a geometry, $\boldsymbol{E}_{0}$ may be approximated by

$\boldsymbol{E}_{0}=\frac{V_{0}}{D_{0}} \frac{\left(u+u^{2}\right)^{1 / 2}}{\log \left[u^{1 / 2}+(1+u)^{1 / 2}\right]^{1 / 2}} \boldsymbol{u}_{z}$

where $V_{0}$ represents the bias voltage and $u=D_{0} / b$; with $D_{0}$ the distance between the substrate and the macroscopic base and $b$ its curvature radius (cf. Fig. 1). In a real experimental configuration both the atomic protuberance fixed on the macroscopic base 
and the adsorbates located in the junction (cf. Fig. 1) generate some distorsion of the initial electric potential distribution $\phi_{0}(\boldsymbol{r})$ associated with $\boldsymbol{E}_{0}(\boldsymbol{r})$. The new potential $\phi(r)$ lying around the adsorbates can be obtained self-consistently by solving the following implicit Fredholm equation:

$$
\phi(r)=\phi_{0}(r)+\phi_{\text {apex }}(r)+\phi_{\text {ad }}(r)
$$
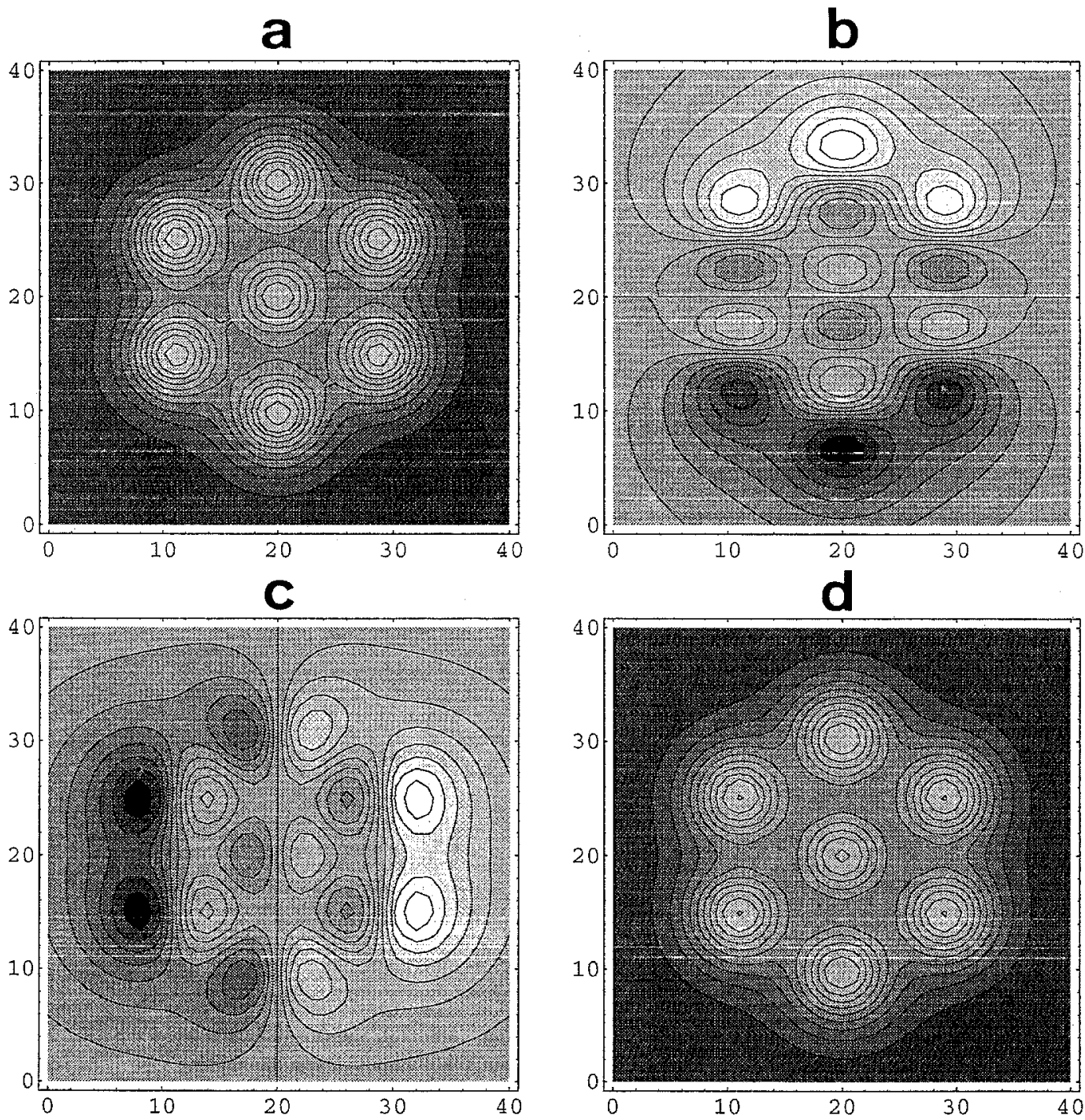

Fig. 2. Grey scale representation of the electric field distribution calculated above a cluster formed of $7 \mathrm{C}_{60}$ molecules adsorbed on a gold surface. The two axes $\mathrm{O} X$ and $\mathrm{OY}$ are labelled in $\AA$. The equilibrium distance between two adjacent molecules is $1 \mathrm{~nm}$ according to recent STM measurements [18]. The calculation is performed in a plane $Z=13 \AA$ parallel to the surface with a tip-sample distance $Z_{0}$ of $30 \AA$. The zeroth order field is along $\mathrm{OZ}$ direction and equal to $0.25 \mathrm{~V} / \AA$ and the contour plots vary by steps of $0.02 \mathrm{~V} / \AA$ : (a) amplitude of the $\mathrm{OZ}$ field component (cf. Fig. (1)). (b) amplitude of the $\mathrm{O} X$ field component. (c) amplitude of the $O Y$ field component. (d) total amplitude of the field. 
where

$$
\begin{aligned}
& \phi_{\text {apex } / \mathrm{ad}}(\boldsymbol{r}) \\
& \quad=\iint K_{0}\left(\boldsymbol{r}, \boldsymbol{r}_{1}\right) \chi_{\mathrm{apex} / \mathrm{ad}}\left(\boldsymbol{r}_{1}, \boldsymbol{r}_{2}\right) \phi\left(\boldsymbol{r}_{2}\right) \mathrm{d} \boldsymbol{r}_{1} \mathrm{~d} \boldsymbol{r}_{2} .
\end{aligned}
$$

In Eq. (2), the last two contributions $\phi_{\text {apex }}(r)$ and $\phi_{\mathrm{ad}}(\boldsymbol{r})$, account for the presence of both the apex and the adsorbates. Moreover $\chi_{\text {apex }}\left(\boldsymbol{r}_{1}, \boldsymbol{r}_{2}\right)$ and $\chi_{\mathrm{ad}}\left(\boldsymbol{r}_{1}, \boldsymbol{r}_{2}\right)$ represent their charge-density susceptibilities [17]. The scalar response function $K_{0}\left(r, r_{1}\right)$ is the electrostatic potential propagator associated with the reference system [17]. Note that in the present description, in which the tip is at tunnelling distance from the substrate, the junction can be assimilated to a weakly leaking capacitor. Consequently, in a first stage, the effect of the tunnelling current on the whole surface charge density of the reference system can be neglected.

In the expressions of $\phi_{\text {apex }}(r)$ and $\phi_{\text {ad }}(r)$, the integrations have to be performed respectively over the volumes of the protrusion and of the adsorbates. The simplest way of solving Eq. (2) is by discretization in the real space. This can be done by using the natural grid formed by the atoms themselves. For this purpose, one can expand the charge-density susceptibilities $\chi_{\text {apex }}\left(\boldsymbol{r}_{1}, \boldsymbol{r}_{2}\right)$ and $\chi_{\mathrm{ad}}\left(\boldsymbol{r}_{1}, \boldsymbol{r}_{2}\right)$ as series of multipolar polarizabilities around the atomic positions $r_{i}$ of the protrusion and $r_{j}$ of the adsorbate. This leads to

$$
\begin{aligned}
\chi_{\mathrm{apex}} & \left(\boldsymbol{r}, \boldsymbol{r}^{\prime}\right) \\
= & \sum_{i=1}^{n}\left\{\alpha_{\mathrm{apex}}^{(1)}[2] \nabla_{r} \delta\left(\boldsymbol{r}-\boldsymbol{r}_{i}\right) \nabla_{r^{\prime}} \delta\left(\boldsymbol{r}^{\prime}-\boldsymbol{r}_{i}\right)\right. \\
& +\frac{1}{9} \alpha_{\mathrm{apex}}^{(2)}[4] \nabla_{\boldsymbol{r}}^{(2)} \nabla_{\boldsymbol{r}^{\prime}}^{(2)} \delta\left(\boldsymbol{r}-\boldsymbol{r}_{i}\right) \\
& \left.\times \delta\left(\boldsymbol{r}^{\prime}-\boldsymbol{r}_{i}\right)+\cdots\right\}
\end{aligned}
$$

and

$$
\begin{aligned}
\chi_{\mathrm{ad}}\left(\boldsymbol{r}, \boldsymbol{r}^{\prime}\right)= & \sum_{j=1}^{m}\left\{\alpha_{\mathrm{ad}}^{(1)}[2] \boldsymbol{\nabla}_{\boldsymbol{r}} \delta\left(\boldsymbol{r}-\boldsymbol{r}_{j}\right) \boldsymbol{\nabla}_{\boldsymbol{r}^{\prime}} \delta\left(\boldsymbol{r}^{\prime}-\boldsymbol{r}_{j}\right)\right. \\
& +\frac{1}{9} \alpha_{\mathrm{ad}}^{(2)}[4] \boldsymbol{\nabla}_{\boldsymbol{r}}^{(2)} \boldsymbol{\nabla}_{\boldsymbol{r}^{\prime}}^{(2)} \delta\left(\boldsymbol{r}-\boldsymbol{r}_{j}\right) \\
& \left.\times \delta\left(\boldsymbol{r}^{\prime}-\boldsymbol{r}_{j}\right)+\cdots\right\}
\end{aligned}
$$

where $\alpha_{\text {apex }}^{(\mathrm{p})}$ and $\alpha_{\mathrm{ad}}^{(\mathrm{p})}$ define the static multipolar polarizabilities of the atoms forming the apex and the adsorbates respectively [12]. The symbol $[k]$ indicates a total contraction of $k$ th order. In a first stage, and with a good accuracy [12], the multipolar contributions higher than the dipolar one can be neglected. This leads to a single summation over the atomic positions. In this approximation, the number of discretization points used to solve the integral Eq. (2) is equal to the total number of atoms. The self-consistent field $E(r)=-\nabla \phi(r)$ occurring in the gap is then given by the following equation

$$
\begin{aligned}
E(r)= & E_{0}(r)+\sum_{i=1}^{n} S\left(r, r_{i}\right) \alpha_{\mathrm{apex}}^{(1)} E\left(r_{i}\right) \\
& +\sum_{j=1}^{m} S\left(r, r_{j}\right) \alpha_{\mathrm{ad}}^{(1)} E\left(r_{j}\right)
\end{aligned}
$$

with

$S\left(r, r^{\prime}\right)=\nabla_{r} \nabla_{r^{\prime}} K_{0}\left(r, r^{\prime}\right)$.

Note that in the present study, the spatial localization of the system (protrusion + adsorbate) allows us to solve Eq. (6) exactly. Indeed, both indices $n$ and $m$ are always finite, so that the resulting matrix equation can be solved by a standard linear algebra procedure. Moreover, in Eq. (7) $\boldsymbol{S}\left(\boldsymbol{r}, \boldsymbol{r}^{\prime}\right)$ represents the electrostatic field propagator associated with the reference system. For a pair of bare metallic electrodes, this dyadic tensor can be derived by applying the procedure described in Ref. [17].

We will focus our attention on a standard system for STM: fullerene molecules adsorbed on a metallic surface [18-20]. We consider a (110) oriented copper tip apex brought to the proximity of an hexagonal aggregate of seven $\mathrm{C}_{60}$ molecules adsorbed on a gold surface. For ideal $\mathrm{C}_{60}$ molecules, the spatial arrangement of carbon atoms can be found in Ref. [21] with atomic polarizabilities extracted from data for graphite. The field propagator $S$ has been constructed from the data of Ref. [12] and for $\alpha_{\text {apex }}^{(1)}$ we have used the value of $9.2 \AA^{3}$ given by Teadrout and Pack [22].

In Fig. 2, a grey scale representation of the electrostatic field distribution is presented. In this first application the STM tip is placed far away from the $\mathrm{C}_{60}$ molecules. The calculation of the field is performed in a plane $Z=13 \AA$ parallel to the substrate. The computational window is limited to a square of side $40 \AA$ centered around the fullerene island. The 
equilibrium distance between two adjacent $\mathrm{C}_{60}$ molecules is set to $1 \mathrm{~nm}$ according to recent STM measurements [18]. In this application the zeroth order applied field (i.e. in the absence of the molecules) is equal to $0.25 \mathrm{~V} / \AA$. In the four different maps $E_{z}(X, Y), E_{x}(X, Y), E_{y}(X, Y)$ and $|E(X, Y)|$ given in Fig. 2, one remarks that the $\mathrm{C}_{60}$ molecules induce a strong confinement of the initial field $\boldsymbol{E}_{0}$. In fact the lateral variation of the total field is directly related to the shape of the molecules themselves; especially for the normal component $E_{z}(X, Y)$ of the field (Fig. 2a) as well as for its modulus (Fig. 2d). Concerning the two parallel components $E_{x}(X, Y)$ and $E_{y}(X, Y)$, one observes a succession of clear and dark regions revealing a depolarization phenomenon. A similar depolarization
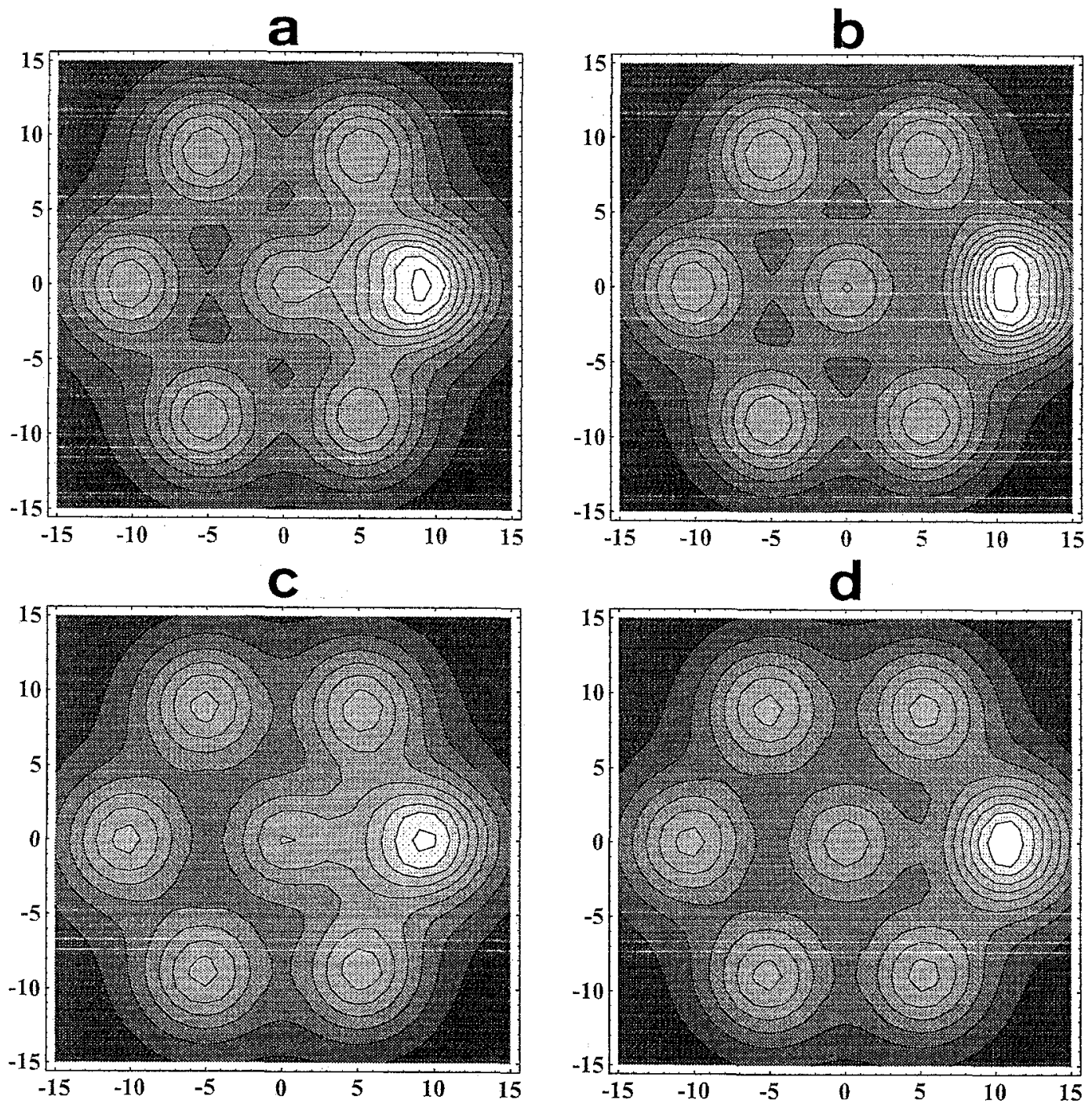

Fig. 3. A sequence of grey scale representations of the variation of the modulus of the electric field calculated in the presence of the tip. The sample is the same as the one considered in cf. Fig. 2. The applied voltage is equal to $4 \mathrm{~V}$ and the contour plots vary by step of $0.025 \mathrm{~V} / \AA$. The black level corresponds to about $0.222 \mathrm{~V} / \AA$ : Various tip-sample configurations are considered: (a) $Z_{0}=16 \AA, Y_{0}=0$, and $X_{0}=7 \AA$. (b) $Z_{0}=16 \AA, Y_{0}=0$, and $X_{0}=11.5 \AA$. (c) $Z_{0}=17 \AA, Y_{0}=0$, and $X_{0}=7 \AA$. (d) $Z_{0}=17 \AA, Y_{0}=0$, and $X_{0}=11.5 \AA$. 
effects also occur above the central $\mathrm{C}_{60}$ molecule of the fullerene island (see Fig. 2d).

When the electrode bearing the metallic protrusion is brought close to the sample, the previous electrostatic field distribution (see Fig. 2) is locally modified by the presence of the apex. A simulation of this operating mode is displayed in Fig. 3 with a tip-apex composed of two atomic layers. The comparison between Fig. 2 and Fig. 3 clearly indicates the presence of an overconfinement introduced by the tip-apex itself. The lateral extension of this peak is very sensitive to the tip-sample distance. When the tip is approaching the sample, the field magnitude increases under the apex and its lateral extension is reduced. For a distance $Z_{0}=16 \AA$ the enhancement factor $\eta=|E| /\left|E_{0}\right|$ reaches about 2.24 . In the application illustrated by Fig. 3 the black level corresponds to $0.222 \mathrm{~V} / \AA$ and the computational window was limited to a square of side $30 \AA$ centered around the hexagonal cluster. Finally, we would like to emphasize that the charge distribution changes inside each $\mathrm{C}_{60}$ molecule is included owing to the self-consistent condition described above (cf. Eq. (2)).

\section{Field induced manipulation of fullerenes}

When the probing tip is scanned above an isolated fullerene molecule adsorbed on the surface, the electric field induces an energy $U\left(\boldsymbol{R}_{0}, \boldsymbol{R}_{\text {ful }}\right)$ which depends on the spatial position of the tip. The vector $\boldsymbol{R}_{\text {ful }}$ defines the position of the center of the fullerene with respect to the surface frame. This energy can be split into two parts

$$
\begin{aligned}
U\left(\boldsymbol{R}_{0}, \boldsymbol{R}_{\mathrm{ful}}\right)= & -\frac{1}{2} \sum_{i=1}^{n} \alpha_{\mathrm{apex}}^{(1)} E^{2}\left(\boldsymbol{r}_{i}, \boldsymbol{R}_{0}, \boldsymbol{R}_{\mathrm{ful}}\right) \\
& -\frac{1}{2} \sum_{j=1}^{m} \alpha_{\mathrm{ad}}^{(1)} E^{2}\left(\boldsymbol{r}_{j}, \boldsymbol{R}_{0}, \boldsymbol{R}_{\mathrm{ful}}\right) .
\end{aligned}
$$

In these relations $E\left(\boldsymbol{r}_{i}, \boldsymbol{R}_{0}, \boldsymbol{R}_{\mathrm{ful}}\right)$ represents the modulus of the self-consistent field at each atomic site. These fields are obtained by solving the set of $(n+m)$ linear equations associated with relation (6). Their implicit dependence with respect to the geometry of the system, described by the two vectors $\boldsymbol{R}_{0}$ and $\boldsymbol{R}_{\text {ful }}$, is accounted for by the electrostatic field propagators. Note that the present treatment allows to include the mutual interaction between the adsorbate and the metallic protrusion.

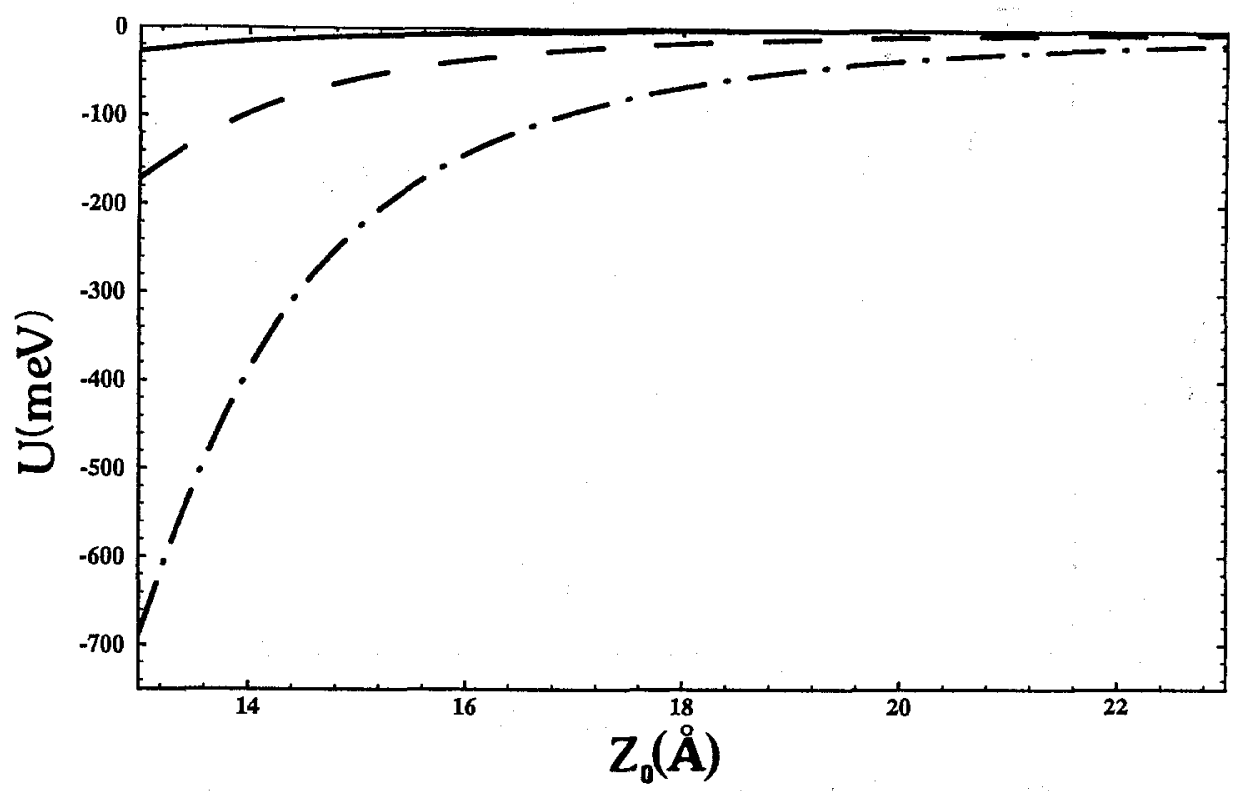

Fig. 4. Variation of the attractive inductive energy $U\left(0,0, Z_{0}\right)$ experienced by a $\mathrm{C}_{60}$ molecule located under the STM tip, as a function of the applied voltage $V_{0} \cdot(\longrightarrow) \mathrm{V}_{0}=1 \mathrm{~V},(---) V_{0}=2.5 \mathrm{~V},\left(\cdot{ }^{\prime} \cdot \cdot\right) V_{0}=5 \mathrm{~V}$. 
In Fig. 4, the variation of the inductive energy $U$ is presented for different bias voltages, as a function of the tip-sample distance $Z_{0}$. In this application we have restricted our study to the case of a single $C_{60}$ molecule adsorbed on Au(110). This system has been extensively studied in STM but no experimental data is available for the equilibrium configuration of a single $\mathrm{C}_{60}$ on the (110) face of Au. Recently the equilibrium position was estimated by using the MM2 molecular mechanics package [23]. For the (110) face of gold this method leads to an equilibrium distance $D_{\text {eq. }}=6.2 \AA$ above the hollow site with an adsorption energy $U_{0}=1.09 \mathrm{eV}$. It may be seen on Fig. 4, that for $V_{0}<1 \mathrm{~V}$, the induction energy is very weak compared to the adsorption energy. Moreover, in the present application, where there is no permanent multipolar moment on the adsorbate, the resulting force associated with $U\left(\boldsymbol{R}_{0}, \boldsymbol{R}_{\text {ful }}\right)$ is always directed towards the tip. Finally one remarks that for stronger applied voltages (for example $V_{0}=5 \mathrm{~V}$ ), the inductive energy can reach $700 \mathrm{meV}$ when the tip is brought at the immediate proximity of the $\mathrm{C}_{60}$.

We now address the problem of field induced manipulation of an isolated $\mathrm{C}_{60}$ molecule adsorbed on the (110) face of gold. According to recent MM2 calculations [23], the variation of the adsorption energy $U_{\text {ad }}$ along the [110] rows of this metallic surface can be fitted by the following relation:

$$
\begin{aligned}
U_{\mathrm{ad}}\left(X_{\mathrm{ful}}\right)= & -\left(U_{0}-\frac{1}{2} U_{\text {cor }}\right) \\
& -\frac{1}{2} U_{\text {cor }} \cos \left(\frac{2 \pi X_{\text {ful }}}{A}\right),
\end{aligned}
$$

where $A$ represents the lattice length of the gold surface along the [110] rows. The magnitude $U_{\text {cor }}$ of the corrugation energy experienced by the molecule along the same direction reaches about $150 \mathrm{meV}$.

The size of a $\mathrm{C}_{60}$ is large compared to the lattice parameter ( $A=4.07 \AA$ for gold). Hence, if we are able to apply, with the STM tip, a sufficiently confined field to locally annihilate the diffusion barrier, it would be possible to precisely manipulate the fullerene molecule. The feasibility of this process can be investigated by studying the variation of the total energy given by

$U_{\mathrm{t}}\left(X_{\mathrm{ful}}\right)=U_{\mathrm{ad}}\left(X_{\text {ful }}\right)+U\left(X_{\text {ful }}\right)$

as a function of the lateral position $X_{\text {ful }}$ of the fullerene and for different values of the tip height $Z_{0}$. A simulation of this operating mode is presented

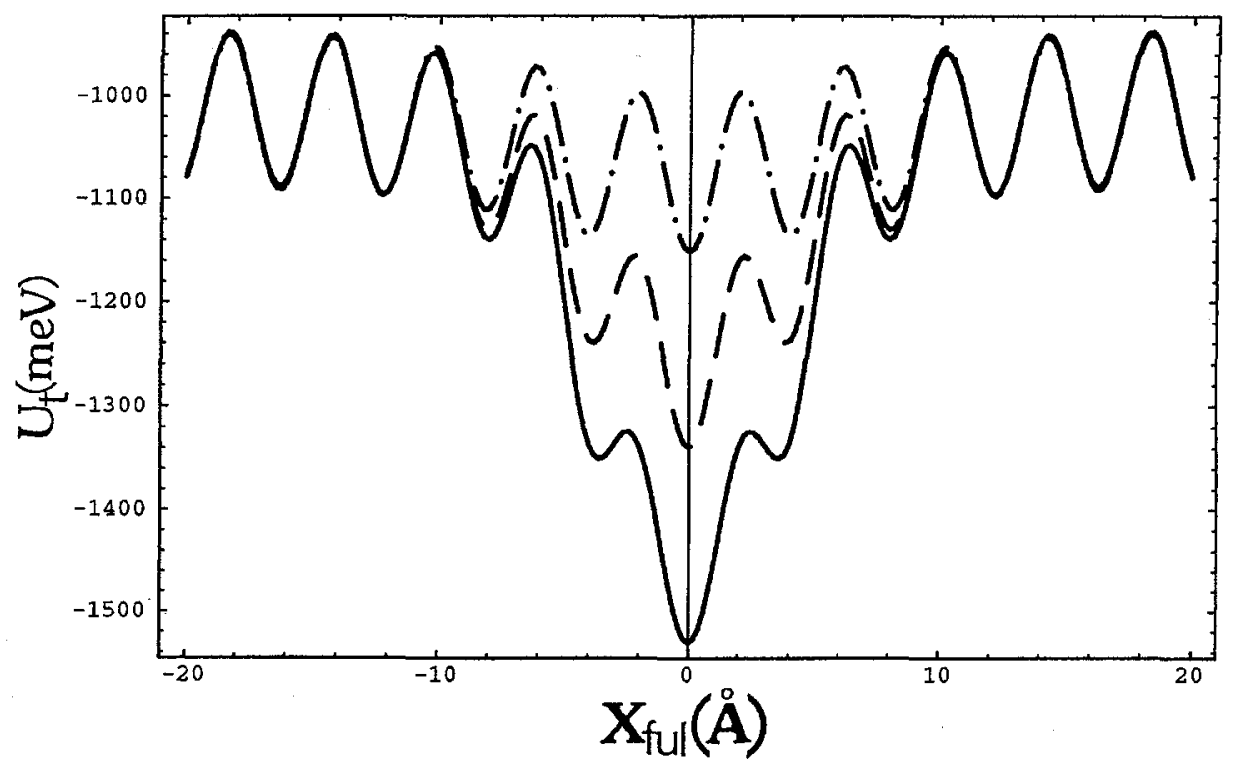

Fig. 5. Variation of the lateral binding energy $U_{t}\left(X_{\text {ful }}\right)$ of a single $\mathrm{C}_{60}$ along a [110] row of a gold surface. The applied voltage $V_{0}$ is equal to $4 \mathrm{~V}$ and the corrugation energy is simulated using data given in Ref. [23]. Three different tip heights $Z_{0}$ are considered: ( $-\longrightarrow$ ) $Z_{0}=13 \AA$, $(---) Z_{0}=14 \AA,(\cdot-\cdot-\cdot) Z_{0}=17 \AA$. 
in Fig. 5 where the applied voltage is kept constant $\left(V_{0}=4 \mathrm{~V}\right)$. As expected, the metallic protrusion induces a depression of weak lateral extension with a depth that strongly depends on the tip-sample distance. For example it may be observed that around $13 \AA$, the barrier heights associated with the two equilibrium sites located apart from the origin are considerably reduced, so that at room temperature the molecule is able to jump from an equilibrium site to another site. Thus, if the tip is moved along the atomic row under these conditions $\left(Z_{0}=13 \AA\right.$ and $V_{0}=4 \mathrm{~V}$ ), the $\mathrm{C}_{60}$ molecule which is trapped by the electrostatic well will follow the tip-apex. Along the [110] direction a similar controllable motion is also possible. But due to the weaker diffusion barrier along the $[\overline{110}]$ atomic rows, the threshold voltage is found to be around $3.25 \mathrm{~V}$ for the same tip-sample distance $Z_{0}=13 \AA$. Finally, we believe that a realistic simulation of the field induced desorption process of an isolated adsorbed $\mathrm{C}_{60}$ molecule could be performed by adding in the present scheme the everpresent tip-sample van der Waals interaction effect. Such extensions are in progress.

\section{References}

[1] D.M. Eigler and E.K. Schweizer, Nature 344 (1990) 524.

[2] D.M. Eigler, P.S. Weiss, E.K. Schweizer and N.D. Lang, Phys. Rev. Lett. 66 (1991) 1189.
[3] D.M. Eigler, C.P. Lutz and W.E. Rudge, Nature 352 (1991) 600.

[4] I.W. Lyo and Ph. Avouris, J. Chem. Phys. 93 (1990) 4479.

[5] J.S. Foster, J.E. Frommer and P.C. Arnett, Nature 331 (1988) 325.

[6] L.J. Whitman J.A. Stroscio, R.A. Dragoset and R.J. Celotta, Science 251 (1991) 1206.

[7] J.A. Stroscio and D.M. Eigler, Science 254 (1991) 1319.

[8] P. Zeppenfeld, C.P. Lutz and D.M. Eigler, Ultramicroscopy 42-44 (1992) 128, and references therein.

[9] X. Bouju, C. Joachim, C. Girard and P. Sautet, Phys. Rev. B 47 (1993) 7454.

[10] T.T. Tsong, Phys. Rev. 44 (1991) 13703.

[11] H.J. Kreuzer, L.C. Wang and N.D. Lang, Phys. Rev. B 45 (1992) 12050; N.D. Lang Phys. Rev. B 45 (1992) 13599.

[12] C. Girard, C. Joachim, C. Chavy and P. Sautet, Surf. Sci. 282 (1993) 400.

[13] Vu Thien Binh and J. Marien, Surf. Sci. 202 (1988) 1319.

[14] H.W. Fink, IBM J. Res. Dev. 30 (1986) 460.

[15] D. Atlan, G. Gardet, Vu Thien Binh, N. Garcia and J.J. Saenz, Ultramicroscopy 42 (1992) 154.

[16] T.T. Tsong, in: Atom-Probe Field Ion Microscopy (Cambridge University Press, Cambridge, 1990), and references therein.

[17] C. Girard, J. Chem. Phys. 85 (1986) 6750.

[18] E.I. Altman and R.J. Colton, Surf. Sci. 279 (1992) 49.

[19] S. Behler, H.P. Lang, S.H. Pan, V. Thommen-Geiser, H.-J. Güntherodt, Z. Phys. B 91 (1993) 1.

[20] R. Berndt, R. Gaisch, J.K. Gimzewski, B. Reihl, R.R. Schlittler, W.D. Schneider and M. Tschudy, Science 262 (1993) 1425.

[21] M. Froimowitz, J. Comput. Chem. 12 (1991) 1129.

[22] R.R. Teadrout and R.T. Pack, Atomic Data 3 (1971) 195.

[23] C. Chavy, C. Joachim and A. Altibelli, Chem. Phys. Lett. 214 (1993) 569. 